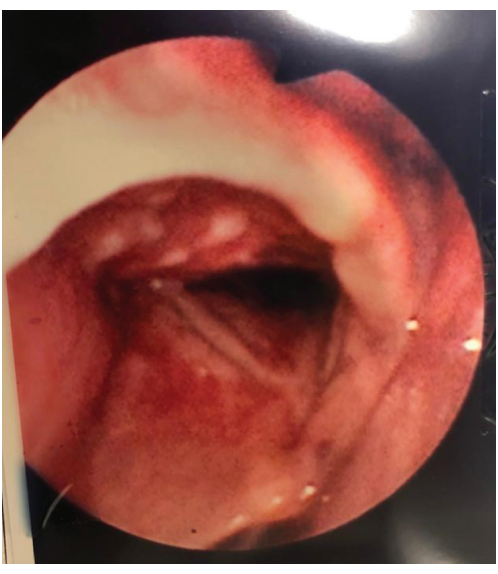

Abstract AB0632B Figure 1: View of the larynx on endoscopy showing the suspicious nodules of granulomatous inflammation

Disclosure of Interests: None declared

DOI: 10.1136/annrheumdis-2019-eular.8260

\section{Scleroderma, myositis and related syndromes}

\section{AB0633 FECAL CALPROTECTIN IN PATIENTS WITH SYSTEMIC SCLEROSIS}

Nafiseh Abdolahi ${ }^{1}$, Mehrdad Aghaie $^{2}$, Haleh Hedayat Mofidi ${ }^{2}$, Mohammad Hadi Gharib ${ }^{3} .{ }^{1}$ Golestan Rheumatology Research center, Gorgan, Iran (Islamic Republic of); ${ }^{2}$ Golestan Rheumatology Research Center, Gorgan, Iran (Islamic Republic of); ${ }^{3}$ Golestan Rheumatology Research Cennter, Gorgan, Iran (Islamic Republic of)

Background: Systemic sclerosis (SSc) is a connective tissue disease with heterogeneous symptoms and a non-predictable process. The most involved organ after the skin is the gastrointestinal tract (Gl tract) and any part of it can be affected. The calprotectin level is increased in plasma, urine, sputum and feces when an inflammatory process is occurring with the presence of neutrophils which is measurable.

Objectives: So we decided to examine fecal calprotectin as a simple method to diagnose $\mathrm{Gl}$ disorders and disease activity in SSc.

Methods: Totally 46 patients with SSc were invited by telephone, who have been referred to sayyad shirazi Hospital and the rheumatologists offices in Gorgan and their information has been registered there. Seven patients did not enter our study because of having the items in exclusion criteria like Diabetes, a history of Gl operation and having other connective tissue diseases at the same time.for the other 39 patients who was remained, a questionnaire about GI symptoms (UCLA SCTS 2.0) was filled in and then their skin was examined and the skin score was determined (the score more than 14 was considered as positive). Blood sample was taken from all patients to do tests as ESR,CRP, Magnesium, Albumin, Ferritin, Folic Acid, Vitamin B12 and Zinc and use them as a scale for malnutrition. Then the stool samples were collected to measure the calprotectin level. At last to determine $\mathrm{Gl}$ disorders in a non-aggressive way, Barium Swallow (Esophagus malfunction) and CT Enterography (intestinal disorders) was done. The patients in our study according to the median of age which was 42, was divided in to two groups: young and middle aged. Due to the fact that FC level wasn't less than 50 $\mu \mathrm{gr} /$ $\mathrm{gr}$ in any of our cases, the patients was divided in to two groups: the one with FC level less than $200 \mu \mathrm{gr} / \mathrm{gr}$ and the one with FC level as $200 \mu \mathrm{gr} / \mathrm{gr}$ or more than $200 \mu \mathrm{gr} / \mathrm{gr}$ and the latter was considered as positive.

Results: As we didn't have any male patients, the correlation between FC level and sex was not verifiable. Increased levels of FC didn't have any correlation with age $(P$ Value $=0.79)$, disease type $(P$ Value $=0.59)$, small intestine wall thickening $(P$ Value $=0.10)$, esophageal dilatation $(P$ Value $=0.18)$, skin score $(P$ Value $=0.25)$, final $G$ l score $(P$ Value $=0.30)$ and none of serum tests. One of the most important reasons, was low sample size.In our study just 5 cases had evidence of wall thickening in CT Enterography and all that 5 cases had increased levels of calprotectin so we see that if we have a Irager sample size we may ensure the connection between these two variables. Also as most of our patients take vitamin and mineral supplements, we couldn't find any signs of micronutrient deficiencies and any correlation with FC level as well.

Conclusion: Although there was no statistical correlation between FC level and variables, the results are in favor of FC's specificity for assessing intestinal wall thickening.

\section{REFERENCES}

[1] -Marie I, Leroi AM, Menard JF, Levesque H, Quillard M, Ducrotte P. Fecal calprotectin in systemic sclerosis and review of the literature. Autoimmunity reviews. 2015;14(6):547-54.

[2] - Andreasson K, Scheja A, Saxne T, Ohlsson B, Hesselstrand R. Faeca calprotectin: a biomarker of gastrointestinal disease in systemic sclerosis. J Intern Med. 2011;270(1):50-7.

[3] - Affandi AJ, Radstake TR, Marut W. Update on biomarkers in systemic sclerosis: tools for diagnosis and treatment. Seminars in immunopathology. $2015 ; 37(5): 475-87$

Disclosure of Interests: None declared DOI: 10.1136/annrheumdis-2019-eular.7663

\section{AB0634 FINDINGS OF EXERCISE ULTRASOUND CARDIOGRAPHY IN PRE-PAH PATIENTS WITH SYSTEMIC SCLEROSISARE CORRELATED WITH EXTENT OF CAPILLARY LOSS DETECTED BY NAILFOLD VIDEOCAPILLAROSCOPY}

Konomi Ashihara, Shusaku Fukaya, Tatsuaki Naganawa, Meiko Hoshino, Yasuchika Kato, Shunji Yoshida, Hidekata Yasuoka. Fujita Health University School of Medicine, Toyoake, Japan

Background: Systemic sclerosis (SSc) is characterized by excessive fibrosis, injuries in vasculature, and production of autoantibodies (1). Patients with SSc are complicated with various organ involvements, which can give a great impact on their prognosis (2). Of these, pulmonary arterial hypertension $(\mathrm{PAH})$ is one of leading causes of death and the determinant of prognosis in SSc (3). And early diagnosis is an important key to improve their prognosis. The nailfold videocapillaroscopy (NVC) is a noninvasive modality which can evaluate the status of microvasculature of patients with SSc. However, the significance of NVC in patients with "pre-clinical" PAH was unclear.

Objectives: To examine the significance of detection of microvascular injury by NVC in patients with pre-clinical PAH patients with SSc.

Methods: Patients with SSc who newly visited the outpatient clinic at Fujita Health University Hospital between August 2015 to March 2016 were consecutively involved. All the patients fulfilled the 1980 preliminary classification criteria for SSc (4). The diagnosis of PAH was based on the definition in 2015 ESC/ERS guideline. The NVC (Medcap ${ }^{\circledR} 3.0$, DS MEDICA) was used to detect microvascular change and average capillary density and the number of capillaries/view were calculated. Also, existence of giant capillaries, hemorrhage and patterns of capillary changes were also documented. Transthoracic ultrasound cardiography (UCG) was performed using iE33 with $5 \mathrm{MHz}$ sector probe S5-1 (Philips). Exercise loading was done with an ergometer (Lode). Ejection fraction, E/e', velocity of tricuspid regurgitation jet (TR jet), tricuspid annular plane systolic excursion (TAPSE) were examined in a supine position. Tricuspid regurgitation pressure gradient (TRPG) was calculated using velocity of TR jet based on Bernoulli principle. Exercise loading was started at 25 Watt, and then increased 25 Watt every 3 minutes and TRPG at maximum exercise was also calculated. Parameters of NVC were compared with those of UCG. Patients with "pre-clinical" PAH were defined as patients 1) asymptomatic, 2) who did not meet the definition of $\mathrm{PH}$ by right heart catheterization $(\mathrm{RHC}), 3)$ whose probability of $\mathrm{PH}$ is lower than "intermediate" by UCG, 4) who did not see any UCG signs for PH based on the guideline and 5) who did not have severe interstitial lung disease, especially $\%$ FVC> $70 \%$ and/or area of ILD based on CT scan was $<20 \%$ of the entire lung field.

Results: Nineteen patients with SSc were newly visited. Male to female ratio was $4: 15$, mean age $63 \pm 13$ years, and mean disease duration was $138 \pm 109$ months. Ratio of limited skin type was $79 \%$. 37\% were with ILD and $11 \%$ were with $\mathrm{PAH}$ confirmed by $\mathrm{RHC}$. There is no corre lation between TRPG at rest and the density of capillaries. However, there is a significant, negative correlation between TRPG at exercise and the capillary density $(r=0.57, P<0.02)$. Furthermore, the same result was observed even with seventeen "pre-PAH" cases $(r=0.67, \mathrm{P}<$ 0.04). 
Conclusion: Our study suggests that the finding of NVC, especially the extent of capillary loss, is correlated with disease process of $\mathrm{PAH}$, even subclinically, and may predict the progression to $\mathrm{PAH}$ in SSc. Since NVC is non-invasive, NVC can detect earlier PAH candidates and contribute to the improvement of prognosis.

\section{REFERENCES}

[1] Denton CP, et al. Trends Immunol 2005; 26: 596.

[2] Steen VD, et al. Ann Rheum Dis 2007; 66: 940.

[3] Avouac J, et al. J Rheumatol 2010; 37: 2290.

[4] The Subcommittee for Scleroderma Criteria of the American Rheumatism Association Diagnostic and Therapeutic Criteria Committee. Arthritis Rheum 1980; 23: 581

Disclosure of Interests: None declared DOI: 10.1136/annrheumdis-2019-eular.4666

\section{AB0635 SYSTEMIC SCLEROSIS}

Radim Bečvár̆, Hana Štorkánová, Barbora Šumová, Maja Špiritović, Sabina Oreska, Ladislav Šenolt, Michal Tomčík. Institute of Rheumatology, Praha, Czech Republic

Background: Skin fibrosis is a hallmark of systemic sclerosis (SSc). There are no widely accepted biomarkers of skin involvement in this condition. Several serum or plasma markers have been studied in patients with SSc - monocyte chemoattractant protein-1 (MCP-1), chemokine (C-X-C motif) ligand 8 (CXCL8), interleukin-13 (IL-13), and some more recognized such as - platelet derived growth factor (PDGF), transforming growth factor-beta 1 (TGF-beta 1), epidermal growth factor (EGF) and basic fibroblast growth factor (bFGF).

Objectives: The aim of this study was to assess several circulating biomarkers which may be relevant to the fibrosing process and further to correlate the obtained data with clinical indicators specific for SSc skin involvement.

Methods: 59 SSc patients (M/F 9/50; mean age 52.1 years, mean disease duration 6.7 years, 36 patients with limited cutaneous SSc and 23 with diffuse cutaneous SSc. As a control group 36 healthy individuals matched to sex and age were examined. Serum concentrations of bFGF granulocyte-colony stimulating factor (G-CSF), granulocyte-macrophage-colony stimulating factor (GM-CSF), MCP-1, PDGF, IL-8 and 13 were analysed using commercial multiplex kit. The following clinical examinations were performed: modified Rodnan skin score (mRSS), Hand Mobility in Scleroderma Test (assessing hand function) (HAMIS), Cochin Hand Function Scale (hand function) (CHFS), Delta Finger-to-Palm Distance (extension-flexion) (dFTP), Inter-lip Distance (inter-lip), Inter-incisor Distance (inter-incisor), and Mouth Handicap in Systemic Sclerosis Scale (mouth opening) (MHISS). For statistical evaluation Spearman's correlation coefficient was used.

Results: When compared with healthy controls serum concentrations of bFGF $\quad(p<0.001), \quad$ G-CSF $\quad(p<0.0001), \quad$ GM-CSF $\quad(p<0.0001), \quad$ MCP-1 $(p<0.0001) \quad$ IL-8 $\quad(p<0.0001)$, and IL-13 $(p<0.001)$ were significantly elevated in SSc cohort. PDGF levels were increased in SSc patients with only a lower significance $(p<0.01)$. bFGF, G-CSF, MCP-1 and IL-8 levels correlated significantly $(\mathrm{p}<0.05)$ with $\mathrm{mRSS}$ and HAMIS. GM-CSF levels correlated with mRSS and HAMIS and there was only a trend for negative correlation with inter-incisor. The was no correlation of $\mathrm{IL}-13$ and PDGF levels with the evaluated clinical data.

Conclusion: Our results have shown that G-CSF, GM-CSF and IL-8 play a substantial role in SSc fibrosing process. Potential biomarkers as bFGF, G-CSF, MCP-1 and IL-8 correlated with a few clinical indices of SSc skin involvement.

\section{REFERENCES}

[1] Carulli MT, Handler C, Coghlan JG, Black CM, Denton CP. Can CCL2 serum levels be used in risk stratification or to monitor treatment response in systemic sclerosis? Ann Rheum Dis 2008;67:105-9.

[2] Chujo S, Shirasaki F, Kondo-Miyazaki M, lkawa Y, Takehara K. Role of connective tissue growth factor and its interaction with basic fibroblast growth factor and macrophage chemoattractant protein-1 in skin fibrosis. $J$ Cell Physiol 2009;220:189-95.

[3] Fuschiotti P, Larregina AT, Ho J, Feghali-Bostwick C, Medsger TA Jr Interleukin-13-producing CD8+ $T$ cells mediate dermal fibrosis in patients with systemic sclerosis. Arthritis Rheum 2013;65:236-46

[4] Hasegawa M, Fujimoto M, Matsushita T, et al. Serum chemokine and cytokine levels as indicators of disease activity in patients with systemic sclerosis. Clin Rheumatol 2011;30:231-7.

Acknowledgement: This study was supported by research grants $A Z V$ 16-33574A and AZV 16-33542A.

Disclosure of Interests: Radim Bečvář Consultant for: consultancy Actelion, Hana Štorkánová: None declared, Barbora Šumová: None declared Maja Špiritović: None declared, Sabina Oreska: None declared, Ladislav Šenolt Grant/research support from: AbbVie, Consultant for: AbbVie, Bris tol-Myers Squibb, Celgene Corporation, Merck Sharp and Dohme, Novartis, Pfizer, Roche, UCB, Amgen, Takeda, Speakers bureau: AbbVie Amgen, Bristol-Myers Squibb, Celgene Corporation, Eli Lilly, Merck Sharp and Dohme, Novartis, Pfizer, Roche, UCB, Michal Tomčík: None declared DOI: 10.1136/annrheumdis-2019-eular.610

\section{$\mathrm{AB} 0636$ \\ INFLUENCE OF CARDIOVASCULAR RISK FACTORS ON NAILFOLD VIDEOCAPILLAROSCOPY IN THE STUDY OF CONNECTIVE TISSUE DISEASES}

Diego Benavent, Laura Nuño, Gemma Bonilla, Diana Peiteado,

Chamaida Plasencia, Victoria Navarro-Compán, Irene Monjo, Alejandro Villalva,

Sara García-Carazo, Carolina Tornero, Patricia Bogas, Elisa Fernández,

Miguel Bernad, Ana Castilla, Pilar Aguado, Maria-Eugenia Miranda-Carus, Eugenio de Miguel, Alejandro Balsa. Hospital Universitario La Paz, Madrid, Spain

Background: Nailfold videocapillaroscopy is a non-invasive technique used to assess Raynaud syndrome. It is mainly used for the early diagnosis of connective tissue disorders (CTD) such as systemic sclerosis. There is some evidence that capillaroscopy findings may be altered by microcirculation abnormalities in patients with cardiovascular risk factors (CVRF).

Objectives: to analyze the influence of cardiovascular risk factors on naildfold capillaroscopy in patients with Raynaud or suspect of CTD.

Methods: An observational and descriptive study of consecutive patients that underwent a videocapillaroscopy examination for the study of Raynaud syndrome was conducted. A "Capiscope" model videocapilaroscope from Optilia was used, with a fixed magnification of 200x. Examination was made on 8 hand fingers, with 2 images per finger. The patients had to be at least 30 minutes in a fixed warm temperature room and without smoking 1 hour before the performance of the test. The following capillaroscopic parameters were considered: nailfold morphology, capillary loop enlargements, megacapillaries, microhaemorrhages, avascular areas and signs of neoangiogenesis. Demographic information (including age gender and previous diagnosis) and cardiovascular risk factors (including arterial hypertension $(\mathrm{HT})$, diabetes mellitus (DM), dyslipidemia (DL) and smoking habit), were collected. The influence of cardiovascular risk factors on naildfold capillaroscopy was analyzed, using univariate and multivariate logistic regression models, adjusted for possible confounders.

Results: Out of the 136 included patients, 91\% were women. Mean age was $54,6 \pm 18,7$ years. Raynaud syndrome was reported in $83 \%$ patients, with a mean duration of $6,1 \pm 5,7$ years and $12 \%$ of the patients had a previous diagnosis of CTD, including systemic lupus erythematosus $(5 \%)$, systemic sclerosis $(4 \%)$, undifferentiated connective tissue disorder $(2 \%)$ and mixed connective tissue disease (1\%). Regarding CVRF, HT was observed in $25 \%$, DM in $7 \%$, DL in $23 \%$ and past or current smoking habit in $32 \%$. Capillaroscopic findings were: loop enlargements $(81 \%)$, megacapillaries $(30 \%)$, microhaemorrhages $(46 \%)$, signs of neoangiogenesis $(71 \%)$ and avascular areas $(20 \%)$.

Regarding the capillaroscopic pattern, $46 \%$ presented a normal or non specific pattern; $31 \%$ a microangiopathy pattern and $23 \%$ a scleroderma pattern (of which $58 \%$ had an early or active scleroderma pattern and $42 \%$ a late scleroderma pattern). A new diagnosis of CTD was made in 24 patients (18\% of the cohort). In the group of patients without CTD, HT was associated with microhaemorrhages $(\mathrm{p}=0.02)$ and avascular 